\title{
The Darwin Ground-based European Nulling Interferometer Experiment (GENIE)
}

\author{
P. Gondoin ${ }^{a}$, O. Absil ${ }^{c}$, M. Fridlund ${ }^{a}$ \\ C. Erd ${ }^{a}$, R. den $\operatorname{Hartog}^{a}$, N. Rando ${ }^{a}$, \\ A. Glindemann ${ }^{b}$, B. Koehler ${ }^{b}$, R. Wilhelm ${ }^{b}$ \\ A. Karlsson ${ }^{a}$, L. Labadie ${ }^{a}$, I. Mann ${ }^{a}$ \\ A. Peacock ${ }^{a}$, A. Richichi ${ }^{b}$, Z. Sodnik ${ }^{a}$, \\ M. Tarenghi ${ }^{b}$, S. Volonte ${ }^{a}$ \\ ${ }^{a}$ European Space Agency, P.O. Box 299, 2200AG Noordwijk, The Netherlands \\ ${ }^{b}$ European Southern Observatory, D-85748 Garching bei Munchen, Germany \\ ${ }^{c}$ Observatoire de Paris-Meudon, place Jules Janssen, Meudon France
}

\begin{abstract}
Darwin is one of the most challenging space projects ever considered by the European Space Agency (ESA). Its principal objectives are to detect Earth-like planets around nearby stars and to characterise their atmospheres. Within the frame of the Darwin programme, the European Space Agency (ESA) and the European Southern Observatory (ESO) intend to build a ground-based technology demonstrator. Such a ground-based demonstrator built around the Very Large Telescope Interferometer (VLTI) in Paranal will test some of the key technologies required for the Darwin Infrared Space Interferometer. The present paper will describe the objectives and the status of the project.
\end{abstract}

Keywords: Darwin, groundbased interferometry, nulling interferometry

\section{INTRODUCTION}

Understanding the principles and processes that generated the Earth, and allowed the evolution of life forms to take place is a major scientific objective which deserves the utmost attention. In particular, the successful detection of Earth-like planets possessing environments benign to life would answer central questions such as "How unique is the Earth as a planet ?" and "How unique is life in the Universe?". To achieve these objectives, the IRSI-Darwin mission of the European Space Agency (Fridlund 2000, 2002) will survey a large sample of nearby stars and search for Earth-size planets within their "habitable zone". IRSI-Darwin will measure their spectra in order to infer the presence of an atmosphere and search for biomarkers.

Detection of Earth-size bodies circling nearby stars is extremely difficult because of the weakness of the planetary signal emitted within a fraction of an arcsecond from an overwhelmingly bright star. A solar type star outshines an Earth size planet by a factor of more than $10^{9}$ in the visible wavelength range. In the infrared spectral range, where the planet's thermal emission increases and the star's emission decreases, the contrast is still higher than $10^{6}$. Only the planetary signal, a millionth of the stellar light, should remain in the input feed of a spectrograph in order to register a planet spectrum in a reasonable time. To accomplish such an extinguishing of light at the relevant spatial scales, the technique of "nulling interferometry" has been selected for IRSI-Darwin. By applying suitable phase shifts between different telescopes in an interferometric array, destructive interference can be achieved on the optical axis of the system in the combined beam while interference is constructive for small off-axis angles. The principle of nulling interferometry for a simple two telescope Bracewell interferometer (Bracewell 1978) is described in Fig. 1. The equivalent transmission map of the nulling interferometer is a set of interference peaks with a sharp null in the centre. By placing the central star under this null, and adjusting the interferometer baseline to the required angular resolution, planets can be detected in the "habitable zone". The actual shape and transmission properties of the pattern around the central null depend on the configuration and the distance between the telescopes. 

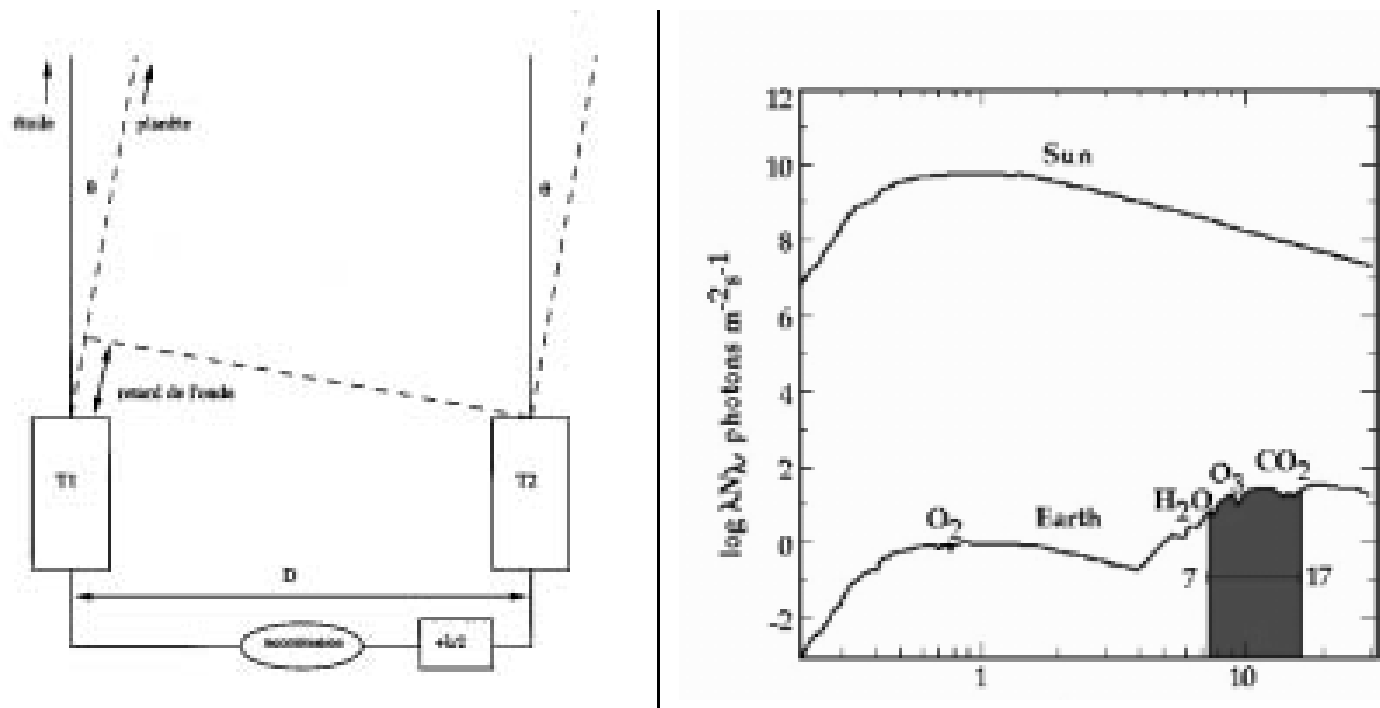

Figure 1. Left: principle of a two telescopes Bracewell interferometer. Right: contrast between the solar flux and the Earth as a function of wavelength in microns. Adapted from Angel et al. (1986).

\section{THE IRSI-DARWIN DEVELOPMENT PROGRAM}

To date, the IRSI-Darwin mission concept (see Fig.2) consists of six $1.5 \mathrm{~m}$ telescopes, each of which is a free flying spacecraft transmitting its light to a central beam-combining unit (see ESA 2000). Using the nulling interferometry technique, the beam combiner could detect and analyze the light from Earth-like planets at interstellar distances of up to 25 parsecs. The mission concept is based on the ability to co-phase telescopes on independent spacecrafts to an accuracy better than $20 \mathrm{~nm}$ and to perform nulling interferometry with a rejection factor of $\approx 10^{6}$ in a wide spectral band extending from 5 to $18 \mu \mathrm{m}$. In-orbit co-phasing of the freeflying telescopes could be performed in successive steps combining first a local radio-frequency positioning system with milli-Newton propulsion devices, then inter-satellite laser metrology with micro-Newton propulsion devices and finally fringe sensors with optical delay lines. These items will be developed within the ESA Technology Research Program (TRP). They will then be tested in-orbit within the frame of the ESA SMART technology demonstration program.

In addition to the co-phasing of free-flying telescopes, a second key issue for IRSI-Darwin is the nulling interferometry technique. Hence, the TRP activities also include the development of Darwin specific optical components, namely achromatic phase shifters, wavefront filtering devices and IR single mode fibers, integrated optics, IR detectors, electronics and coolers, optical delay lines and fringe sensors as well as other components for interferometry. Laboratory nulling breadboards with star-planet simulators are currently designed that will test these components before the end of 2002. These innovative set-up use the narrow telecommunication band around $1.65 \mu \mathrm{m}$ where off-the-shelves components are available. Based on their performance, a follow-up activity has been identified which shall adapt their design to the mid-IR band and test the nulling interferometry technique on astronomical targets in IRSI-Darwin representative operating conditions.

The Very Large Telescope Interferometer (VLTI) at the European Southern Observatory (ESO) is the most appropriate infrastructure on-ground to perform such a test. Hence, ESA and ESO decided to initiate a definition study for a Groundbased European Nulling Interferometry Experiment which shall operate in the central laboratory of the VLTI at Mount Paranal (Chile). This experiment is called Darwin-GENIE.

\section{DARWIN-GENIE: A TECHNOLOGY DEMONSTRATOR}

The primary objective of the Darwin-GENIE nulling experiment is to gain experience on the design , manufacture and operation of a nulling interferometer using IRSI-Darwin representative concept and technology. 

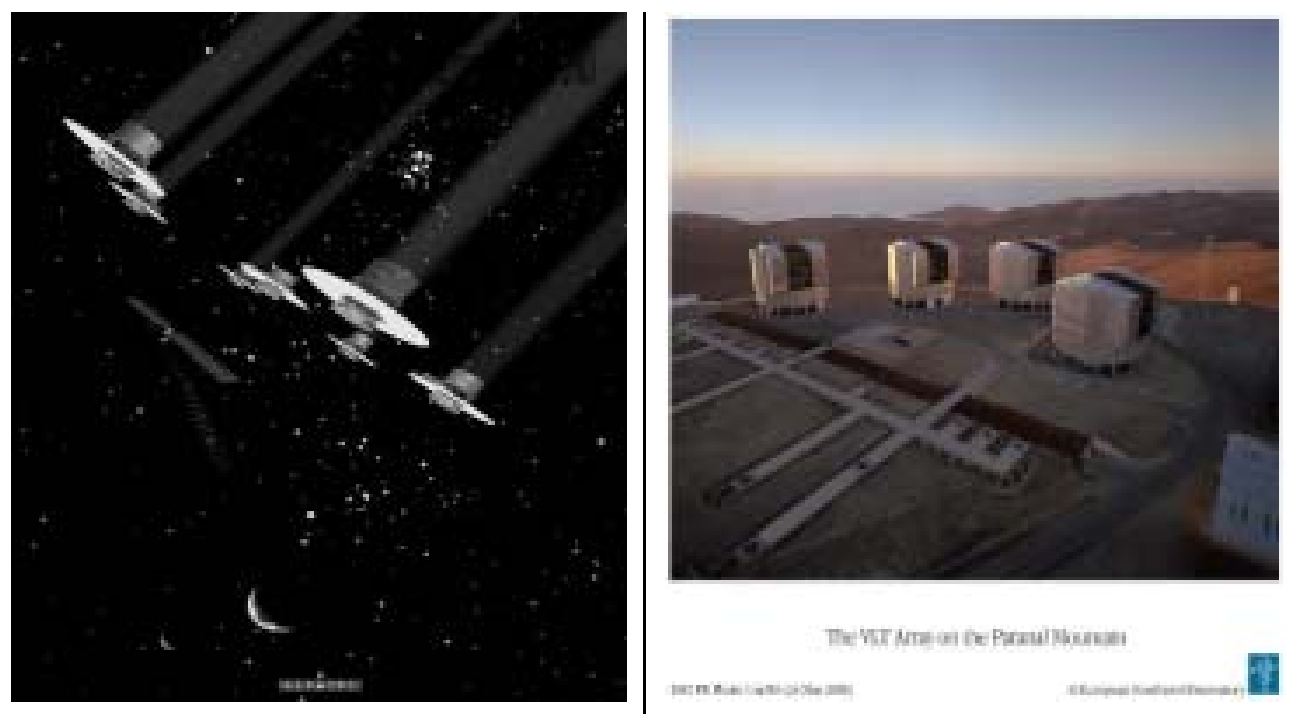

Figure 2. Left: Artist view of the IRSI-Darwin Space Interferometer orbiting at Sun-Earth L2 point (courtesy Alcatel Space Division). Right: the ESO Very Large Telescope Interferometer.

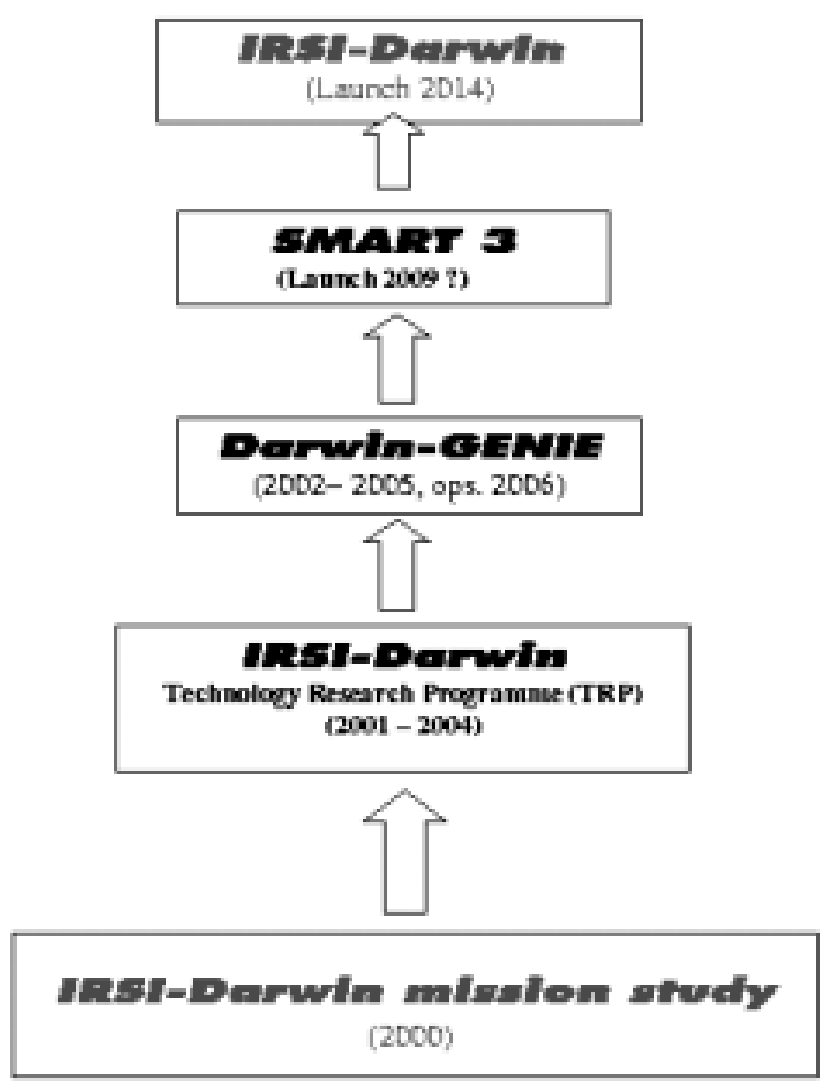

Figure 3. The major steps to Darwin 


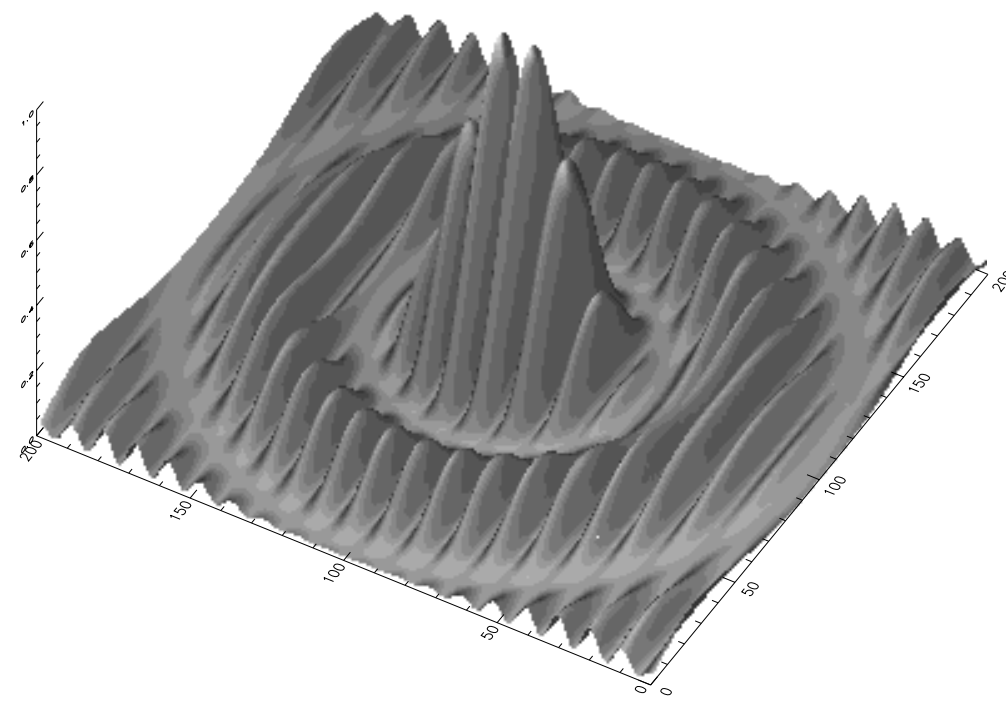

Figure 4. Transmission map of a diffraction limited Bracewell interferometer with circular entrance aperture.

Nulling test with the highest rejection factor on single stars or close binaries in broad mid-IR spectral bands will achieve this objective with the limitations imposed by the turbulence and infrared background of the Earth atmosphere. The Darwin-GENIE experiment will combine all optical functions foreseen into the future Darwin Infrared Space Interferometer. It will benefit from the existing VLTI infrastructure, including the telescopes with chopping and adaptive optics, the delay lines, the fringe sensors and the beam combiner laboratory. The overall performance of the instrument will heavily depend on the performance of all VLTI subsystems and in particular on the adaptive optics and co-phasing subsystems. The GENIE optical bench within the VLTI laboratory will provide the functions specific to the nulling interferometry technique, namely photometry and amplitude control, polarization matching, phase shifting, beam combination and internal modulation, spatial filtering, spectrometry, detection, electronics and cryogeny. The detailed architecture of Darwin-GENIE will be defined a the definition study. It will take into account the ESO VLTI interface chracteristics and the output of the ESA TRP activities.

\section{DARWIN-GENIE: A PREPARATION OF THE DARWIN SCIENCE PROGRAM}

A second objective of GENIE is to prepare the IRSI-Darwin science program through a systematic survey of IRSI-Darwin candidate targets. The solar zodiacal cloud, a sparse disk of $10-100 \mu$ diameter silicate grains, is the most luminous component of the solar system after the Sun. Its optical depth is only $\approx 10^{-7}$, but a patch of the solar zodiacal cloud 0.3 AU across has roughly the same emitting area as an Earth sized planet. Similar and even brighter clouds may be common in other planetary systems and present a severe obstacle for the direct detection of extra-solar terrestrial planets. A systematic survey of IRSI-Darwin candidate targets will screenout those stars for which circumstellar dust prevent the detection of Earth-like planets. Bright exo-zodiacal clouds are easier to detect than extra-solar terrestrial planets, but finding an exo-zodiacal cloud is still difficult. The total emission from our zodiacal cloud is no more than $10^{-4}$ of the Sun's at any wavelength. Photometric surveys like the Infrared Astronomical Satellite (IRAS) survey can only detect exo-zodiacal clouds that are > 500 times as optically thick as the solar clouds (Backman \& Parece 1993). Attempts to spatially resolve faint exo-zodiacal clouds with single-dish telescopes in the mid-infrared and near infrared (Kuchner \& Brown 2000) have not yielded better detection limits. A Bracewell interferometer using two ESO VLTI 8m Unit Telescopes (UT) could provide better performance. 


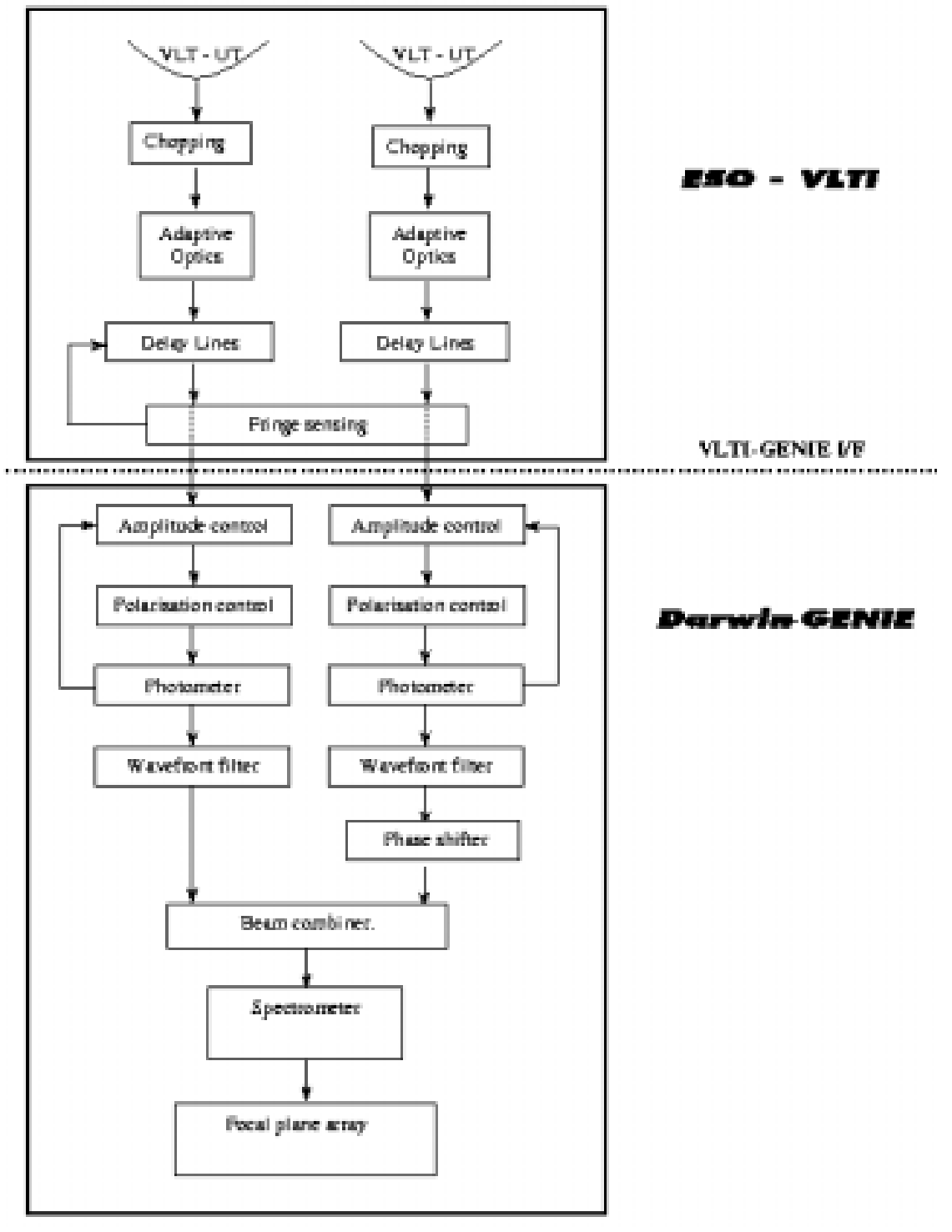

Figure 5. Functional description of GENIE

\subsection{The Bracewell interferometer: a candidate configuration for Darwin-GENIE}

The transmission map $\mathrm{T}(\theta, \phi)$ of a diffraction limited Bracewell interferometer with two circular entrance apertures can be expressed as a function of wavelength, telescope diameter D, and projected baseline B as follows.

$$
T(\theta, \phi)=2 \times\left(J_{1}(\pi \theta D / \lambda) /(\pi \theta D / \lambda)\right)^{2} \times \sin ^{2}(\pi \theta \cos (\phi) B / \lambda)
$$

$\theta, \phi$ are respectively the angular distance to the boresight and the azimut angle in the plan of the sky with respect to the projected baseline. Eq.(1) assumes a diffraction limited operation without any residual wavefront error, with a perfect $\pi$ phase shift between the two arms of the interferometer and equal amplitudes of the recombining fields. In practice, the central null of the transmission map will be degraded by amplitude and optical path differences (OPD) between the two arms of the interferometer that are not perfectly corrected by the amplitude OPD control servo-loops. Also, wavefront distorsions induced by atmospheric turbulence will 


\begin{tabular}{|c|c|c|c|c|}
\hline Spectral band & $\mathrm{K}$ & $\mathrm{L}$ & $\mathrm{M}$ & $\mathrm{N}$ \\
\hline Exo-zodiacal flux (Jy) & $0.2 \times 10^{-3}$ & $0.5 \times 10^{-3}$ & $0.7 \times 10^{-3}$ & $1.1 \times 10^{-3}$ \\
Corresponding magnitude & 16.2 & 14.2 & 13.4 & 11.3 \\
Star/cloud contrast & $1.5 \times 10^{5}$ & $3 \times 10^{4}$ & $1.4 \times 10^{4}$ & $2.5 \times 10^{3}$ \\
\hline
\end{tabular}

Table 1. Exozodiacal flux, star/cloud brightness contrast of an exo- zodiacal cloud 10 times denser the solar zodaical dust and located around a G2 V star at $10 \mathrm{pc}$. The background flux through a UT telescope is also given.

only be partially compensated by the adaptive optics. Spatial filtering of the high frequencies wavefronts errors by monomode optical fibers will be required (Ollivier \& Mariotti 1997). The coupling efficiency inthe fiber will be variable (Shaklan \& Roddier 1988) depending on the fluctuating wavefront and pointing errors. Hence, the envelope of the transmission map of the interferometer will significantly depart from a Bessel function and will be highly time variable.

In the Gaussian approximation, the optimum injection efficiency into a fiber is found for an f/D ratio such that (Ruilier 1998) the diffraction limited image has approximately the size of the core. The geometrical extent of the beam seen by the fiber is then $S \Omega \approx \lambda^{2}$. While the IR thermal background contribution is proportional to $S \Omega$, the signal of the exo-zodiacal dust cloud included into the field-of-view of the interferomter is proportional is the collecting surface. Hence, the ratio signal to noise of an exo-zodiacal dust cloud observed in the mid-IR by a Bracewell interferometer is proportional to its collecting area. The largest telescopes shall be used specially when operating in a spectral band where the background is very high. The output signal of a Bracewell interferometer pointing to a star surrounded by a dust cloud can be expressed.

$$
S(\theta, \phi)=A_{\mathrm{eff}} \times \int_{\theta} \int_{\phi} T(\theta, \phi) \times\left(O_{\mathrm{s}}(\theta, \phi)+O_{\mathrm{z}}(\theta, \phi)\right) \times d \theta \times d \phi+\text { Background }
$$

$T(\theta, \phi)$ is the transmission map of the interferometer degraded by residual amplitude fluctuations and optical path differences between the two interferometer arms. $O_{\mathrm{s}}(\theta, \phi)$ and $O_{\mathrm{z}}(\theta, \phi)$ are the brightness of the star and of the exo-zodiacal dust disk respectively. Background is the incoherent background signal which include the thermal emission from the sky and from the telescope and instrument optics. Since the only reliable observations on zodiacal dust have been carried out in the solar system, spectral properties of the exo-zodiacal dust emission can only be extrapolated from observations of the solar interplanetary dust cloud performed by the Diffuse Infra-Red Background Experiment (DIRBE) on board the the COBE satellite. The most comprehensive model is due to Kelsall et al. (1998). It has been implemented in an IDL package called ZODIPIC (Küchner \& Serabyn 2002). In this model, the dust density $n(r, z)$ is presumed to be in a form that is separable into a radial power law term and a vertical decreasing exponential term, i.e $n(r, z)=n_{0} \times r^{-\alpha} \times f(z / r)$. The parameters are fitted to the DIRBE observations and a multiplicative factor $n_{0}$ is used to parametrized the large range of dust densities that can be expected in exo-zodiacal dust clouds. A disk inner cut-off is assumed to account for the sublimation of dust grain close to the star at temperatures higher than about $1500 \mathrm{~K}$. Since the contribution of the scattered stellar light is negligible in the infrared, the brightness of the cloud is expressed as an integral along the line of sight of the dust thermal emission.

$$
O_{z, \lambda}=\int(1-A) \times E_{\lambda} \times B_{\lambda}(T) \times K_{\lambda}(T) \times d s
$$

$A$ is the albedo, $E_{\lambda}$ an emissivity modidfication factor that measures deviations from the blackbody thermal radiance $B_{\lambda}(T)$, and $K_{\lambda}(T)$ the DIRBE color-correction factor. The temperature $\mathrm{T}$ of the dust is a decreasing function (taken as $T(r)=286 \times r^{-0.467}$ ) of the distance $\mathrm{r}$ to the star. For baselines significantly greater than the diameters of the individual telescopes, Eq.(1) indicates that the first maximum of the transmission map is located approximately at $\theta=\lambda /(2 \times B)$. Due to Earth rotation, the projected baseline B on the plane of the sky will rotate and vary in apparent length and orientation with a period of one day. However, the hot inner dust of nearby solar-like zodiacal clouds, which has a high density, will most contribute to the overall signal 
registered by a $50 \mathrm{~m}$ baseline interferometer. Hence, flux considerations alone indicates that both the L' band at $3.8 \mu \mathrm{m}$ and the $\mathrm{N}$ band around $10.2 \mu \mathrm{m}$ could be appropriate for the detection of exo-zodiacal dust clouds around nearby stars with two ESO VLT telescopes operating as a Bracewell interferometer. In principle, the M band could also be considered but can be highly affected by the presence of varying amount of water vapour in the atmosphere. Table 1 shows the flux of an exo-zodiacal dust cloud 10 times denser than the solar zodiacal dust around a G2 V star at $10 \mathrm{pc}$. The contrast between the star and the dust cloud brightness is also given for the different atmospheric bands. The $\mathrm{N}$ band present a significant advantage over the $\mathrm{L}$ ' band since the contrast between the star and the dust cloud is lower by a factor of 10. Exo-zodiacal dust cloud detection can be achieved with a nulling rejection of a few 1000 in the $\mathrm{N}$ band (compare to a few $10^{4}$ in the L' band). Since in addition the wavelength is longer, requirements on the control accuracy of the optical path difference are much less severe in the $\mathrm{N}$ band.

\subsection{Stellar leakage and thermal background issues}

The transmission map of a Bracewell interferometer exhibits a narrow null with a $\theta^{2}$ transmission on axis. Eq. 2 indicates that the high spatial resolution of a two telescopes interferometer with a $\approx 50$ m baseline does not completely reject the starlight on axis specially when stars are closer than 50 pc. A residual stellar signal $S_{\text {leak }}$ leaks through the transmission map. It can be expressed as a function of the operating wavelength $\lambda$, interferometer baseline $B$ and angular radius $\theta_{\mathrm{s}}$ of the star.

$$
S_{\text {leak }}=A_{\mathrm{eff}} \times \int_{\theta} \int_{\phi} T(\theta, \phi) \times O_{\mathrm{s}}(\theta, \phi) \times d \theta \times d \phi \approx\left(A_{\mathrm{eff}} \times O_{\mathrm{s}}\right) \times\left(\pi^{2} / 8\right) \times\left(\theta_{\mathrm{s}} B / \lambda\right)^{2}
$$

Assuming that the average stellar leakage can be remove by calibration, the major effect of the stellar leakage is to induce important noise contributions. One contribution is the photon noise of the mean stellar leak $<S_{\text {leak }}>$. A second source of noise is the erratic fluctuation $\sigma_{\text {leak }}$ of the stellar leakage due to residual variations at high frequencies of the amplitude and optical path difference between the two arms of the interferometer. Nulling interferometers using three or four ESO VLT telescopes could provide theoretically a broader null and could be therefore less sensitive to stellar leakage. However, such configurations with three or four VLTs would be non-linear. The Earth rotation would induce a non-homothetic deformation of the projected bidimensional array on the plane of the sky, so that amplitude and/or phase variations would be needed to compensate the array deformation during the night and to maintain a broad $\theta^{4}$ null. Additional engineering complexity would come from the increased number of fringe sensors and OPD control units. Furthermore, the stellar light leakage through groundbased nulling interferometer using more than two telescopes would be in practice much larger than their theoretical transmission due to their high sensitivity to residual OPD and Strehl amplitude fluctuations (Absil 2002).

$$
S / N=S_{z} \sqrt{\Delta \lambda \Delta t} / \sqrt{\text { Background }+S_{z}+<S_{\text {leak }}>+\sigma_{\text {leak }}^{2}}
$$

Photon noise and fluctuations of the stellar leakage are major limitations for the detection of exo-zodiacal dust clouds using a groundbased nulling interferometer. Eq.(4) shows that the stellar leakage of a bracewell interferometer and therefore its contributions to the noise can be reduced by operating at long wavelength with short baselines. Hence, the detection of exo-zodiacal dust clouds at VLTI would be most efficient with a Bracewell interferometer operating in the $\mathrm{N}$ band and combining two large $8.2 \mathrm{~m}$ UT telescopes with the shortest VLTI baselines (e.g 46 or 56 meters). Assuming a $20 \mathrm{~nm}$ rms OPD control accuracy, we estimated that an exo-zodiacal dust disk fifteen times brighter than the solar zodiacal cloud could be detected around a G2 V star at $10 \mathrm{pc}$ within $750 \mathrm{~s}$. This detection performance is limited by the IR thermal background noise which is particularly important in the $\mathrm{N}$ band.

In addition to the photon shot noise, the background contributes to the noise by its temporal fluctuations. In the above estimate, we assumed that chopping is efficient enough to remove background fluctuations. In practice, little is known about the stability of the background in the mid-IR both on long and short time scales. For background subtraction, GENIE could benefit from the VLT chopping sub-system where the target 
and background are alternatively observed by tilting the VLT secondary mirrors. Since the background is not expected to be spatially uniform, the nodding technique could be applied. The main drawback of this chopping method is that the tip-tilt unit, the adaptive optics and the fringe sensor cannot remain in close loop since they do not see the central guide star when measuring the background. All these subsystems have to reclose their loops before each single interferometric measurement is made. The overall detection performance of GENIE will thus depend on the efficiency of these subsystems which are expected to close their loops in a few hundreds of milli-seconds, while a chopping period is expected to last typically for $100 \mathrm{msec}$. Alternative methods could improve this chopping efficiency. These include counter chopping of a control beam operating in a separate $\mathrm{H}$ or $\mathrm{K}$ band or a dual feed interferometer where one of the beam remains on the guide star to feed the subsystems. A third possibility is to use an internal chopping method similar to the one proposed by Küchner and Serabyn (2002) in the context of the Keck nuller. In this method, the exit pupils of each of two telescopes are divided in two parts. Each half-pupil of one telescope is recombined destructively with the corresponding half-pupil of the other telescope, such that two Bracewell interferometers with parallel baselines are formed. Their nulled output are then combined with a $\pm \pi / 2$ phase shift. Chopping can thus be carried out at high frequencies by alternately registering the signal from these two outputs. Based on IR thermal background measurement results, the definition study of GENIE will trade-off these different chopping methods and assess the feasibility of detecting exo-zodiacal dust clouds around nearby stars in the $\mathrm{N}$ band with a Bracewell interferometer. The study will establish the analysis methods of the interferometric data and the associated calibration modes of the instrument that are needed to retrieve the circumstellar signal from the residual starlight and to infer informations on the morphology of circumstellar clouds.

\section{DARWIN-GENIE: DIRECT DETECTIONS OF EXO-PLANETS?}

Since 1995, more than 80 extrasolar planets have been discovered as companions to solar-type stars (Marcy, Cochran \& Mayor 2000). All of these planets were discovered indirectly in high-precision Doppler surveys. These surveys measure the radial velocity Doppler shift of the parent star due its periodic motion around the center of mass of the star plus planet system. This technique is most sensitive to massive planets with short orbital periods. The extrasolar planets that have been discovered today have mass ranges from $M \times \sin i \approx$ 0.15 to 10 Jupiter masses (where $i$ is the orbit inclination onto the observer line of sight) with orbital periods ranging from 3 days to a few year. Because of their proximity to the parent star $(<<1 \mathrm{AU})$, the atmosphere of some of these planets is much hotter than that of Jupiter (and of the Earth), hence their name of hot Jupiters. With temperatures sometimes higher than $1000 \mathrm{~K}$, their thermal emission is maximum in the infrared $\mathrm{K}$ and L bands. With baselines ranging from 46 to 130 meters, the VLTI is optimized for the detection in the L band of planets at about 5-15 milli-arcsec from their parent star which correspond to 0.05-0.15 AU for a nearby star at 10 parsecs. This is specially fortunate since these planets are precisely the hot Jupiters of which a significant sample is known. The contrast between a hot Jupiter and its parent star in the L band is reduced to less than $10^{4}$ compare to the $10^{6}$ in the $\mathrm{N}$ band for Earth-like planets.

As previously mentioned, the photon noise and the fluctuations of the stellar leakage induced by residual variations of the amplitude and optical path difference between the two arms of a Bracewell interferometer are major limitations to the detection of faint objects around nearby stars. Eq. 4 shows that the starlight leakage through a two telescopes Bracewell interferometer increases as the square of the baseline and decreases as the square of the wavelength. Hence, the requirements on the OPD control accuracy are much more stringent in the L', band than in the $\mathrm{N}$ band to limit the noise contribution due to stellar leakage fluctuations. This is illustrated in Fig. 6 which shows the ratio signal to noise of a $850 \mathrm{~K}$ Jupiter detection around a G2 dwarf as a function of the star distance. Two $8.2 \mathrm{~m}$ telescopes are assumed to operate in a Bracewell configuration within the $\mathrm{L}$ band. A $0.5 \mu$ bandwidth and a 100 seconds integration time are assumed. The target distance, planet separation and baseline are assumed to be in the optimum condition where the hot Jupiter is located on the first maximum of the Bracewell transmission map. The influence of residual piston error (left curve) and of the amplitude matching error (right curve) are plotted as a function of the star distance assuming no other error contributor. For stars closer than $10 \mathrm{pc}$, the detection performance are limited by the performance of the OPD and amplitude control loops. The performance requirements of these subsystems for bright exoJupiter detection with a Bracewell interferometer consisting of two UTs operating either in the K or in the 
Table 2. Performance requirement for bright exo-Jupiter detection of a Bracewell interferometer with two UTs operating either in the $\mathrm{K}$ or in the $\mathrm{L}$ band

\begin{tabular}{|cc|c|c|}
\hline Performance requirements & (unit) & K band & L band \\
\hline Amplitude matching accuracy & & $<1 \%$ & $<1 \%$ \\
OPD control accuracy & $(\mathrm{nm})$ & $<6 \mathrm{~nm}$ & $<10 \mathrm{~nm}$ \\
Stellar leakage absolute calibration accuracy & & $<0.5 \%$ & $<2.5 \%$ \\
Background subtraction accuracy & & $<510^{-3}$ & $<510^{-5}$ \\
\hline
\end{tabular}
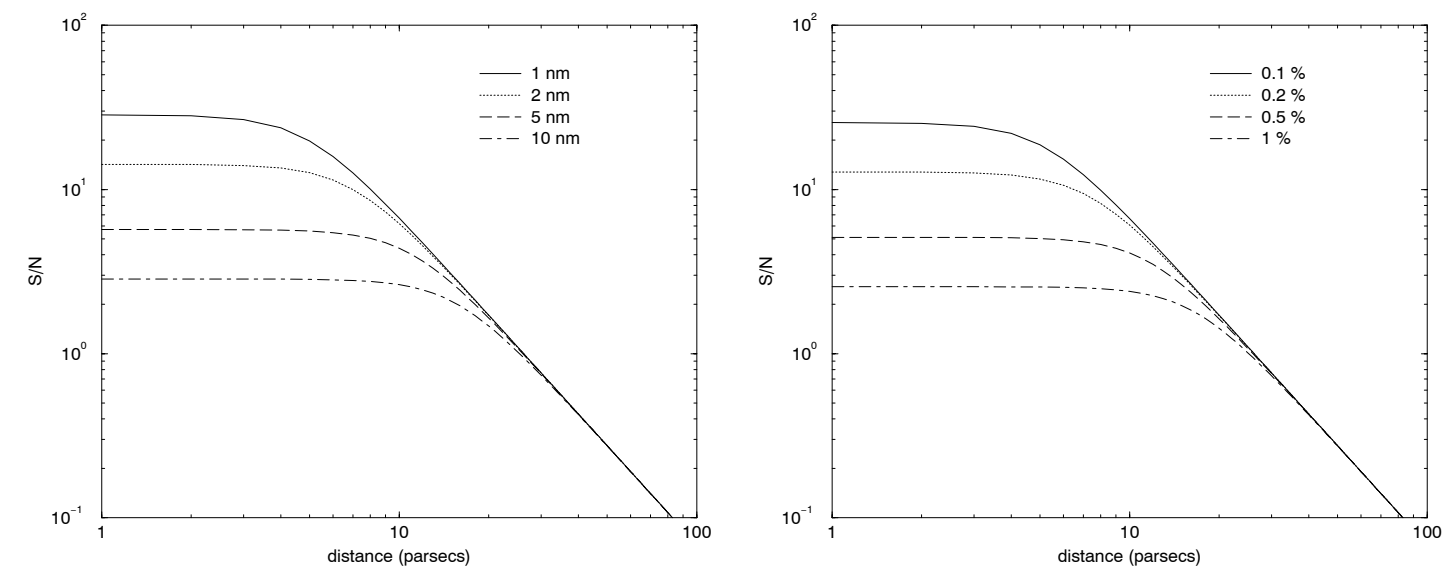

Figure 6. Ratio signal to noise of a $850 \mathrm{~K}$ Jupiter detection around a G2 dwarf as a function of the star distance. Two $8.2 \mathrm{~m}$ telescopes are assumed to operate in a Bracewell configuration within the L band. The target distance, planet separation and baseline are assumed to be in the optimum condition where the hot Jupiter is located on the first maximum of the Bracewell transmission map. The influence of residual piston error (left curve) and of the amplitude matching error (right curve) are plotted as a function of the star distance assuming no other error contributor.

$\mathrm{L}$ band are summarized in Table 2. The requirement on the OPD control accuracy between the two arms of the interferometer are slightly relaxed in the $\mathrm{L}$ band compared with the $\mathrm{K}$ band. They are theoretically within the limitation imposed by the brightness of the reference central star for nearby objects. However, a second OPD control loop operating at high frequencies would be required which performance feasibility is to be demonstrated. As already mentioned, three or four telescopes configurations can in principle achieve a broader null of the star signal. Howerer, in practice, they do not offer performance improvements specially in the $\mathrm{K}$ or $\mathrm{L}$ bands since they are not robust to residual atmospheric OPD fluctuations.

In the L band, the starlight leakage is much higher than the hot-Jupiter signal around nearby stars. Hence, one additional difficulty is to calibrate out this parasitic signal. A double-Bracewell configuration (see Fig. 7) using internal modulation between two Bracewell nulling interferometers could subtract the IR background and the starlight leakage from the overall nulled signal with an accuracy sufficient to retrieve the signal from the brightest exo-Jupiters. The principle of this double Bracewell technique would be to recombine the nulled output of two Bracewell interferometer with a $\pm \pi / 2$ phase shift so that new transmision maps are obtained which are asymmetric with respect to the center of the field of view. A maximum of transmission in one map corresponds to a minimum of transmission in the other map. Therefore, an extended source with central symmetry has the same contribution in both outputs. On the other hand, a hot Jupiter located on the maximum of transmission of one map would not give any signal through the other map. Thus, the Jupiter signal can be modulated and therefore retrieved by alternating the two outputs. Although possible in theory, direct detection of the hottest exoplanets on-ground using this nulling interferometry technique in the L' band would be even more demanding in term of OPD control accuracy than a single Bracewell interferometer. It is however worth noting that three double-Bracewell configurations are possible at VLTI (see Fig. 8) which offer flexibility in adapting 


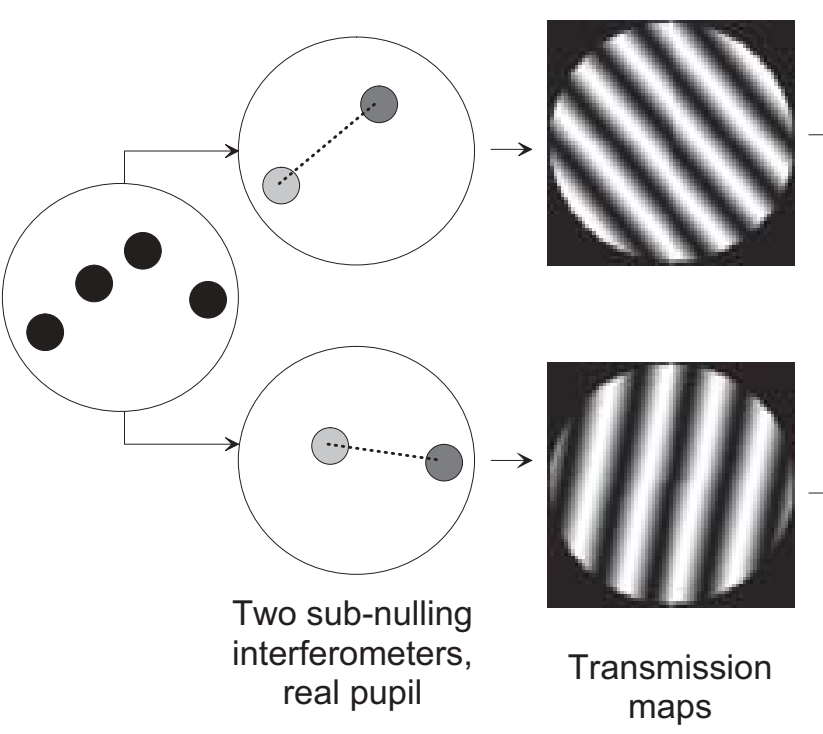

(a)

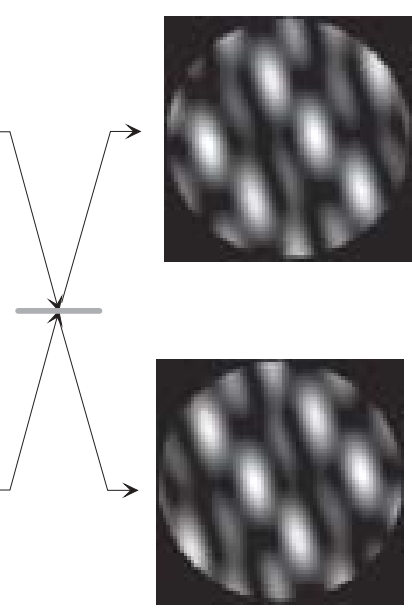

Two asymmetric maps, conjugated by central symmetry

(c)

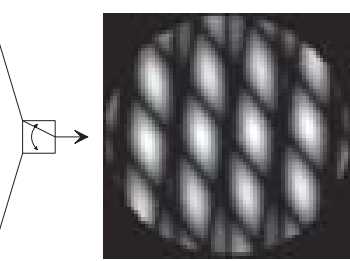

Modulation map

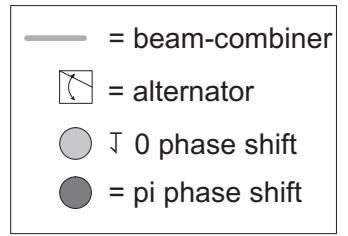

(d)

Figure 7. Principle of internal modulation between two Bracewell interferometers

the transmission map to the orbital position of the exo-Jupiters around their parent star.
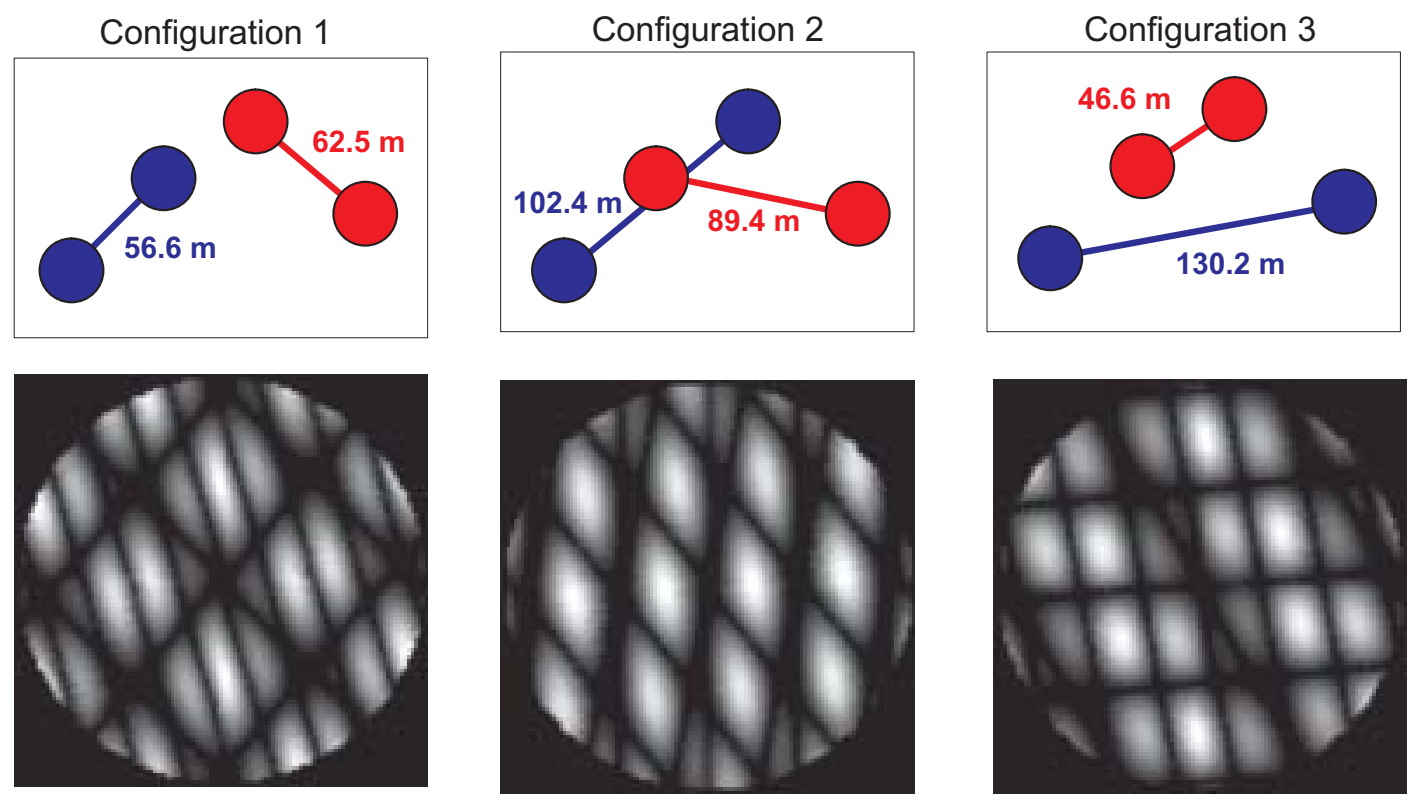

Figure 8. Possible double-Bracewell configurations and associated transmission maps using ESO UTs. 


\section{DARWIN-GENIE: A GENERAL USER INSTRUMENT}

The nulling interferometry technique in the infrared is well suited to spectroscopic studies of any faint cool object located in the immediate environment of a bright astrophysical source (e.g. Voit 1997). A wide range of studies could potentially be conducted using the Darwin-GENIE experiment including infrared spectroscopy of cool dwarfs in spectroscopic binaries, of protoplanetary disks around T Tauri stars and Herbig Haro objects (Malbet \& Bertout 1995). Such programs would be important scientific by-products of the Darwin-GENIE experiment.

\section{SUMMARY}

Darwin is one of the most challenging space projects ever considered by the European Space Agency (ESA). Its principal objectives are to detect Earth-like planets around nearby stars and to characterise their atmospheres. Within the frame of the Darwin programme, the European Space Agency (ESA) and the European Southern Observatory (ESO) intend to build a ground-based technology demonstrator. This Groundbased European Nulling Interferometry Experiment (GENIE) shall operate in the central laboratory of the VLTI at Mount Paranal (Chile). The experiment will combine all optical functions foreseen into the future Darwin Infrared Space Interferometer. It will benefit from the existing VLTI infrastructure, including the telescopes with chopping and adaptive optics, the delay lines, the fringe sensors and the beam combiner laboratory. The GENIE optical bench within the VLTI laboratory will provide the functions specific to the nulling interferometry technique, namely amplitude and polarization matching, phase shifting, beam combination, spatial filtering, internal modulation, spectrometry, detection and cryogeny.

The prime objective of Darwin-GENIE is to gain experience on the design , manufacture and operation of a nulling interferometer. The ESO Very Large Telescope Interferometer offers a unique opportunity to fulfill this objective which feasibility is guaranteed. Nulling test with the highest rejection factor on single stars or close binaries in broad mid-IR spectral bands will achieve this objective with the limitations imposed by the turbulence and infrared background of the Earth atmosphere. Secondary objectives of Darwin-GENIE are to prepare the IRSI-Darwin science program through a systematic survey of IRSI-Darwin candidate targets, to perform Darwin related science achievable from ground and to provide the science community with a scientifically useful instrument. ESA and ESO will initiate an initial definition study to be conducted by European Industries and Scientific Institutes. The study will establish a preliminary design of the instrument including the specifications of its subsystems and a description of their implementation using technologies developed within the frame of the ESA Technology Research Program. Among the different solutions that will be investigated, a candidate design is a Bracewell interferometer operating in the $\mathrm{N}$ band and combining two large $8.2 \mathrm{~m}$ UT telescopes with the shortest baseline (e.g 46 or 56 meters) available at VLTI. Based on IR thermal background measurement results, the definition study of GENIE will trade-off different chopping methods including classical chopping and nodding technique, counter chopping or internal modulation and assess the feasibility of detecting exo-zodiacal dust clouds around nearby stars with a Bracewell interferometer. Upon completion of the definition study, ESA and ESO will decide whether to proceed in the manufacturing and test of a Darwin Groundbased European Nulling Interferometer Experiment. 


\section{REFERENCES}

1. Absil O, 2002. Genie: study of aperture configurations, technical report

2. Angel J.R., Cheng A.Y., Woolf N.J., 1986, Nature 322, 341

3. Backman D.E., Parece F., 1993, in Protostar and Planet III, ed. E.H. Levy \& J.I. Lunine (Tucson, Univ. of Arizona Press), 1253

4. Bracewell R.N., 1978, Nature 274, 780

5. ESA, 2000. Darwin: the Infrared Space Interferometer: Concept and Feasibility Study Report ESA$\mathrm{SCI} / 2000 / 12$

6. Fridlund M., 2000, in Proceedings of the Conference 'Darwin and Astronomy - the Infrared Space Interferometer', Stockholm, Sweden, 17-19 November 1999, ESA SP-451

7. Fridlund M., 2002, in Proceedings of the Conference 'Stellar Structure and Habitable Planet Finding' Cordoba, 11-15 June 2001, ESA SP-485

8. Kelsall T., Weiland J., Franz B., et al. 1998, ApJ 508, 44

9. Küchner M. J., Brown M.E., 2000, PASP 112, 827

10. Küchner M. J., Serabyn E. 2002, ApJ submitted

11. Malbet F., Bertout C., 1995, A\&AS, 113, 369

12. Marcy G.W., Cochran W.D., Mayor M., 2000, in Protostar and Planet IV, ed. V. Mannings, A.P. Boss \& S.S. Russel (Tucson, Univ. of Arizona Press), 1285

13. Ollivier M., Mariotti J.M., 1997, App. Opt. 365340

14. Ruilier C., 1998, SPIE 3350, 319

15. Shaklan S., Roddier F., 1988, App. Opt. 27, 2334

16. Voit M. G., 1997, ApJ 487, L109 